\section{Infections et agents infectieux}

Ce numéro de médecines/sciences dirige le projecteur sur plusieurs agents infectieux vedettes. Le virus de l'hépatite $C$ est aujourd'hui l'un des virus (sinon LE virus) les plus menaçants pour l'homme.

L'interféron pernnet de ralentir ou de stopper l'évolution de l'hépatite, mais les échecs restent nombreux.

La séquence d'une protéine virale non structurale semble jouer un rôle essentiel dans la sensibilité à cette thérapeutique. Le virus HTLV1 est également très prévalent, au moins dans certaines régions, et peut entraîner diverses affections, leucémies ou syndromes neurodégénératifs dans la physiopathologie desquels interviendrait la protéine Tax. On sait maintenant que celle-ci se lie à une protéine cellulaire Int-6 normalement colocalisée avec la protéine PML dans les corps nucléaires. De la même manière que PML est délocalisée dans les leucémies promyélocytaires avec translocation $t(15 ; 17)$, Int-6 est délocalisée sous l'effet de la synthèse de Tax dans les cellules infectées par HTLV1, ce qui pourrait représenter une piste quant à la physiopathologie de cette infection. Concernant l'agent des maladies à prions, les informations restent rares et nous ne pouvons que noter avec espoir les projections indiquant que l'épizootie de BSE en Grande-Bretagne touche à sa fin. Quant au VIH, dont la réplication est efficacement bloquée par des antiprotéase virales, on apprend qu'il pourrait utiliser ses protéases pour inactiver la protéine antiapoptotique Bcl2, hâtant l'apoptose des cellules infectées et leur élimination par des macrophages qui seraient ainsi à leur tour contaminés.

\title{
Un nouveau facteur prévisionnel de réponse à l'interféron au cours de l'hépatite chronique $C$ : la variabilité de la région NS5A
}

Les facteurs prévisionnels de réponse au traitement par l'interféron sont de mieux en mieux connus. Parmi les facteurs de mauvaise réponse, on distingue ceux liés à la maladie (ancienneté de l'hépatite $\mathrm{C}$, présence d'une cirrhose, niveaux de GGT et de ferritinémie élevés), les facteurs liés au sujet (sexe masculin, sujet âgé, déficit immunitaire, consommation excessive d'alcool), les facteurs liés au traitement (dose et durée insuffisantes), et les facteurs liés au virus de l'hépatite $\mathrm{C}$ ( $\mathrm{VHC}$ ) lui-même, qui semblent les plus importants [1]. Parmi ces paramètres virologiques, l'influence du niveau de virémie et celle du génotype sont les mieux établies. Ces constatations suggèrent que la variabilité génomique du VHC est impliquée dans la réponse au traitement par l'interféron, et qu'elle pourrait influencer le niveau de réplication virale. Plus récemment, il a été démontré que l'infection simultanée par plusieurs souches de VHC appartenant au même génotype, les quasi-espèces, constituait un facteur de mauvaise réponse au traitement [2]. On suppose que les mutations dans la région E2/NS1 du VHC, aboutissant aux quasi-espèces, sont la conséquence d'une pression de sélection exercée par la réponse immunitaire de l'hôte et sont probablement le mécanisme principal mis en jeu dans le passage à la chronicité de l'hépatite chronique C. De cette constatation, l'idée que l'interféron puisse lui-même, par son effet "immunomodulateur", influencer l'émergence de mutants du VHC ne pouvait qu'éclore. Mais une idée encore plus simple devait être exploi- tée, et ce qu'a fait l'équipe d'Enomoto (Tokyo, Japon) dans un article paru dans le Journal of Clinical Investigation [3]. Cette idée est la suivante: chez les patients non répondeurs, la persistance de VHC à la fin du traitement n'exclut pas que certaines souches aient pu être sensibles au traitement. Ainsi, si l'on met en évidence des souches qui disparaissent au cours d'un traitement par l'interféron, celles-ci sont probablement sensibles au traitement, alors que celles qui émergent ou persistent sont, par définition, résistantes au traitement interféron. En comparant la séquence des souches de VHC sensibles et résistantes ainsi définies, il devait être possible de définir le mécanisme génétique associé au phénotype "résistance au traitement". Les auteurs ont étudié 3 patients non répondeurs, infectés par le type $1 b$, chez lesquels plusieurs souches étaient présentes simultanément au cours du traitement, ce qui pouvait être mis en évidence par la technique de SSCP (single strand conformational polymorphism) après amplification de la région hypervariable du VHC. Chez ces 3 patients, il était possible de mettre en évidence une souche, initialement présente, qui disparaissait au cours du traitement. Les auteurs ont dans un premier temps comparé l'intégralité de la séquence de cette souche "sensible " à celle de la souche "résistante" majoritaire détectée en fin de traitement. Ils ont ainsi, chez les 3 patients, constaté que les 2 souches avaient une région NS5A systématiquement différente, la différence entraînant des changements d'acides aminés dans la partie 
carboxyterminale de la protéine NS5A. (La fonction de cette protéine n'est pas connue; la polymérase virale est codée par la région NS5B.) Les autres régions non stucturales, la capside, et les régions 3'NC et 5'NC n'étaient pas systématiquement différentes. Dans un second temps, les auteurs ont comparé les séquences de la région NS5A chez 6 nouveaux patients non répondeurs et 9 patients répondeurs, infectés par des souches de VHC de type 1b: il existait, chez tous les patients répondeurs, des mutations touchant les codons 2209 à 2248, par rapport à la souche "sauvage " HCV-J, prototype du génotype $\mathrm{lb}$, alors que tous les patients non répondeurs étaient infectés par des souches conservées par rapport à la souche HCV-J. Les mutations les plus fréquentes concernaient le codon 2218, faisant disparaître un résidu histidine de la protéine NS5A. Les auteurs définissaient cette région de $40 \mathrm{AA}$ la "région déterminant la sensibilité au traitement interféron". Ces mutations n'ont pas été recherchées pour des souches de génotype différent de $1 \mathrm{~b}$.

Dans un second article, paru dans le New England Journal of Medicine [4], les auteurs ont ensuite évalué si la séquence NS5A (2209-2248), déterminée avant traitement, pouvait prédire la réponse à l'interféron. Quatre-vingtquatre patients ont été inclus dans cette étude rétrospective. Ces patients, tous infectés par des souches de type $1 b$, avaient été traités pendant 6 mois par 6 à $10 \mathrm{MU}$ d'interféron, 3 fois par semaine. Il s'agissait soit d'interféron $\alpha$ recombinant (60 patients), soit d'interféron lymphoblastoïde (24 patients). La réponse complète à l'interféron était définie par la persis- tance 6 mois après l'arrêt du traitement de transaminases normales et d'ARN du VHC indécelable par PCR intriquée (nested PCR), réalisée tous les 3 mois. 21 patients étaient répondeurs complets et 63 non répondeurs. Les séquences ont été déterminées dans la région NS5A par séquençage direct, technique qui ne peut mettre en évidence que les séquences majoritaires. L'influence de la présence de mutations dans cette région sur la réponse à l'interféron a été testée en analyse multivariée en association avec l'âge, le sexe, le mode de contamination (transfusion ou non), l'ancienneté de l'hépatite $\mathrm{C}$, la dose totale et le type d'interféron reçus, le niveau de transaminases, le degré de fibrose sur la biopsie hépatique, et le niveau de virémie préthérapeutique. L'analyse des séquences a permis la répartition des souches en trois groupes : un groupe "sauvage" (30 patients), où la séquence correspondait à celle de la souche HCV-J, un groupe «mutant» (16 patients), caractérisé par la présence d'au moins quatre mutations sens, et un groupe "intermédiaire» (38 patients), caractérisé par la présence d'une à trois mutations sens. Une réponse complète était observée chez $100 \%$ des patients du groupe "mutant", contre $0 \%$ du groupe "sauvage" ( $p<0,001)$ et $13 \%$ du groupe «intermédiaire» $(p<0,001)$. La réponse au traitement était significativement associée à un niveau de virémie plus faible $\left(0,5 \cdot 10^{6} \mathrm{Eq} / \mathrm{ml}\right.$ vs $\left.4,4 \cdot 10^{6} \mathrm{Eq} / \mathrm{ml}, \mathrm{p}<0,001\right)$. Il existait de plus une relation entre le niveau de virémie et le type de séquence NS5A: chez les "mutants", le niveau de virémie était plus faible que chez les "sauvages " ou les "intermédiaires " $\left(<0,5 \cdot 10^{6} \mathrm{Eq} / \mathrm{ml}\right.$ comparé à $4,7 \cdot 10^{6} \mathrm{Eq} / \mathrm{ml}$, $\mathrm{p}<0,001$ ou à $\left.4,0 \cdot 10^{6} \mathrm{Eq} / \mathrm{ml}, \mathrm{p}<0,001\right)$. Toutefois, le niveau de virémie n'était pas systématiquement impliqué dans la relation entre la variabilité de la région NS5A et la réponse à l'interféron : trois répondeurs complets étaient infectés par une souche "mutante» et avaient une virémie élevée alors que six patients non répondeurs, infectés par une souche "sauvage" avaient une virémie faible. En analyse multivariée, seul le nombre de mutations dans la région NS5A était associé à la réponse à l'interféron (odds ratio 5,3, p=0,007). Les mécanismes mis en jeu dans cette relation demeurent inconnus. Ils pourraient concerner la réplication virale qui serait altérée en cas de mutations, ou modifier une région cible pour des protéines antivirales induites par l'interféron.

V.D.M.

1. Marcellin P, Pouteau M, Boyer N, Castelnau C. Benhamou JP, Erlinger $\mathrm{S}$. Facteurs de réponse au traitement par l'interféron $\alpha$ chez les malades atteints d'hépatite (: chronique. Perspectives thérapeutiques. Gastro Enterol Clin Biol 1995; 19: 9911000 .

2. Kanazawa Y, Hayashi N, Mita E, Li T, Hagiwara A, Fusamoto $H$, Kamada $T$. Influence of virus quasi species on effectiveness of interferon therapy in chronic hepatitis $\mathrm{C}$ patients. Hepatology $1994 ; 20: 1121-30$.

3. Enomoto N, Sakuma 1, Asahina Y, Kurosaki M, Murakami T, Yamamoto C, Izumi N, Marumo F, Sato C. Comparison of full-length sequences of interferon-sensitive and resistant hepatitis ( : virus Ib. Sensitivity to interferon is conferred by amino acid substitutions in the NS5A region. J Clin Invest 1995 ; 96 : 224-30.

4. Enomoto N, Sakuma I, Asahina Y, Kurosaki M, Murakami T, Yamamoto C, Ogura Y, Izumi N, Marumo F, Sato (.. Mutations in the nonstructural protein 5A gene and response to interferon in patients with chronic hepatitis $C$ virus $1 \mathrm{~b}$ infection. N Engl J Med 1996; 334: 77-81.

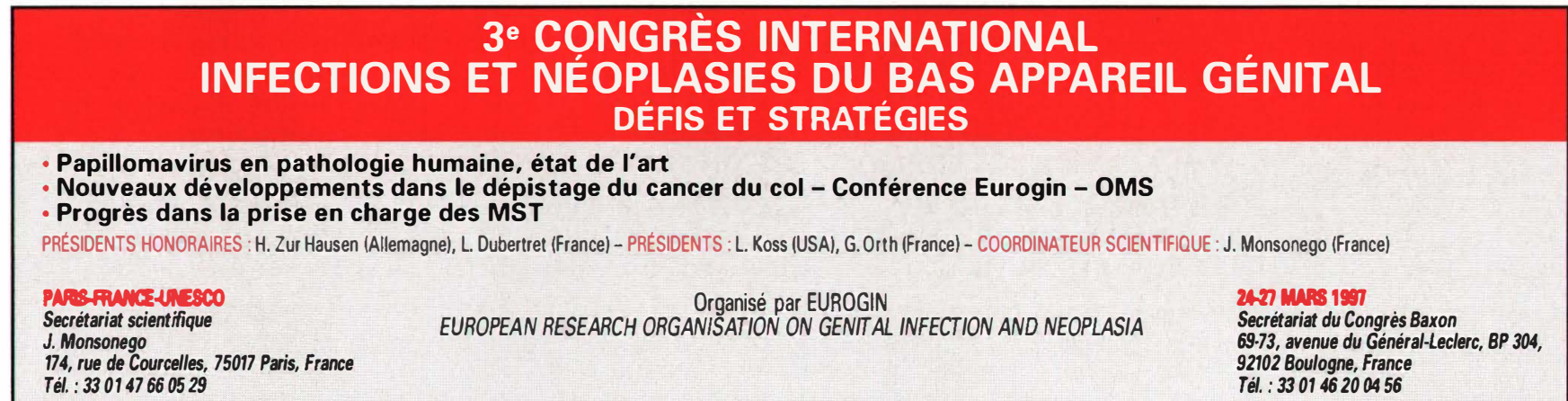

